\title{
Achados necroscópicos em cães e gatos vítimas de intoxicação exógena*
}

\section{Necroscopic findings in dogs and cats victim of intentional intoxication}

\author{
Tália Missen Tremori, ${ }^{* *}$ Sérvio Túlio Jacinto Reis, ${ }^{* \star, * \star *}$ Mara Rita Rodrigues Massad, ${ }^{* *}$ Laila Massad Ribas, ${ }^{* \star}$ \\ Luiz Mauricio Montoya Flórez, ${ }^{* *}$ Noeme Sousa Rocha**
}

\begin{abstract}
Resumo
O reconhecimento adequado dos sinais clínicos e lesões anatomopatológicas são importantes para caracterizar os casos de intoxicação exógena em cães e gatos. O diagnóstico definitivo do agente tóxico deve ser realizado por exame toxicológico. No período de 2009 a 2014 foram analisados 42 casos de necropsia com histórico de intoxicação exógena de 31 (73,8\%) cães e 11 $(26,2 \%)$ gatos. Destes $21(50 \%)$ apresentaram Boletim de Ocorrência e $22(52,4 \%)$ foram submetidos ao exame toxicológico. A maior prevalência verificada foi intoxicação por carbamato e nos exames necroscópicos a principal causa de morte insuficiência cardiorrespiratória, seguida por choque hipovolêmico. Na histopatologia do fígado, rim e cérebro as principais lesões encontradas foram congestão, degeneração e hemorragia. Os órgãos apresentaram sinais de autólise e putrefação. Com base no estudo observou-se que as técnicas diagnósticas complementares, histopatologia e exame toxicológico, fornecem suporte adicional na elaboração de laudos em casos de intoxicações exógenas criminais envolvendo animais.
\end{abstract}

Palavras-chave: Medicina forense, patologia forense, legislação veterinária, patologia veterinária.

\begin{abstract}
Appropriate recognition of the clinical signs, lesions anatomical pathological that characterizes cases of animals intoxication that take to death. Associated with the methods of identification laboratorial of forensic toxicology is fundamental to establish a definitive diagnosis of the toxic agent. In period 2009 to 2014 are selected 42 cases, $31(73,8 \%)$ dogs and $11(26,2 \%)$ cats. These cases $21(50 \%)$ feature police reports and $22(52,4 \%)$ are made toxicological exam. Highest prevalence was intoxication for carbamate. Necroscopic exam revealed that main cause of death were cardiac respiratory insufficiency and hypovolemic shock. In histopathology of liver, kidney and brain the main lesions are congestion, degeneration and bleeding. The organs showed signs of autolysis and putrefaction. The diagnostic techniques used, histopathology and necropsy are additional and helps veterinarian to make reports for litigation in cases of intentional poisoning in animals.
\end{abstract}

Keywords: Forensic medicine, forensic pathology, veterinary legislation, veterinary pathology.

\section{Introdução}

A conscientização crescente da sociedade atual leva ao incremento da legislação relativa à proteção animal, em áreas como conservação do meio ambiente, combate ao contrabando, tráfico ilegal de animais e bem-estar animal, neste condão a medicina veterinária legal, é uma especialidade que vem ganhando espaço desde o final do século $X X$, trata-se de ciência forense de alta complexidade, que utiliza conhecimentos médicos para o cumprimento das leis de caráter técnicocientífico, cuja finalidade é a elucidação de crimes envolvendo animais (Cooper e Cooper, 2008; Mcdonaugh et al., 2015).

Dentro os tipos de crimes notificados e cometidos contra os animais de companhia, a intoxicação exógena é comum. $E$ de acordo com estudos anteriores, as intoxicações fatais têm como causa principal os pesticidas, sendo $50,4 \%$ causadas por carbamatos, $18,9 \%$ por rodenticidas anticoagulantes, $5,1 \%$ por organofosforados e 3,4\% por rodenticidas não anticoagulantes (Wang et al.; Xavier et al., 2007; Medeiros et al., 2009).

A intoxicação de animais enquadra-se entre as condutas vedadas pela Lei de Crimes Ambientais, Lei no 9.605/98, previsto no Artigo 32, onde cita que praticar ato de abuso, maus-tratos, ferir ou mutilar animais silvestres, domésticos ou domesticados, nativos ou exóticos, é crime e tem como pena detenção de três meses a um ano, além da multa (Brasil, 1998; 2014).

Para elucidação de provas em processos judiciais envolvendo intoxicação de animais é elaboração de documentos de caráter técnico científico por profissional médico veterinário devidamente capacitado, neste caso um perito, ou também conhecido como expert (Munro, 1998; Tremori e Rocha, 2014; Mcdonough et al., 2015).

*Recebido em 15 de fevereiro de 2017 e aceito em 22 de julho de 2018.

**Universidade Estadual Paulista, UNESP, Faculdade de Medicina Veterinária e Zootecnia, Departamento de Clínica Veterinária, Botucatu, SP, Brasil.

${ }^{* * *}$ Polícia Federal, Departamento da Polícia Federal, Curitiba, PR, Brasil.

Autor para correspondência: talia_missen@hotmail.com. 
A comprovação deste tipo de crime depende do reconhecimento adequado dos sinais clínicos, lesões anatomopatológicas características dos casos de intoxicação que levam á óbito associados com a identificação laboratorial por exame toxicológico, esse conjunto de análises são fundamentais para estabelecer um diagnóstico definitivo do agente tóxico (Gwaltney-Brant, 2007; Cooper e Cooper, 2008; Byard e Boardman, 2011).

A toxicologia forense busca esclarecer, com finalidade legal, a presença ou ausência de um agente tóxico no organismo, no sentido de auxiliar na investigação de crimes, bem como o andamento do processo judicial (Below e Lignitz, 2003; Bulcão et al., 2010).

Nos casos onde existe suspeita de intoxicação, a identificação do agente é de grande importância. Quando um animal vem a óbito, para um diagnóstico preciso é ideal a realização da análise anatomopatológica associada ao exame toxicológico, aumentando assim a possibilidade de confirmação dos casos suspeitos (Cummings et al., 2011; Klaassen et al., 2013).

A realização do exame necroscópico é muito importante e tem papel fundamental no esclarecimento dos fatos e na determinação da causa mortis do animal, pois através dos achados de necropsia pode-se suspeitar o agente causador. A histopatologia forense pode auxiliar no diagnóstico de intoxicações, com a análise minuciosa dos tecidos (Cummings et al., 2011; Mcdonough et al., 2015).

\section{Material e métodos}

Foram analisados durante o período de cinco anos, compreendido entre 2009 a 2014, exames necroscópicos realizados no Laboratório de Patologia Veterinária e Medicina Legal da Faculdade de Medicina Veterinária e Zootecnia - Universidade Estadual Paulista - Unesp - Campus de Botucatu. O presente estudo possui aprovação do Comitê de Ética no Uso de Animais (CEUA), sob o protocolo número 122/2014.

As espécies eleitas foram cães (Canis lupus familiares) e gatos (Felix catus), de ambos os gêneros, independente de raça e idade. Como critérios de inclusão cada animal deveria apresentar indícios de intoxicação criminal segundo a anamnese e realização de exame necroscópico convencional documentado.

Além disso, de cada caso foram coletadas informações a respeito da confirmação do vestígio pela toxicologia e a realização ou não de documento elaborado pela Autoridade Judiciária (Boletim de Ocorrência).

Os cadáveres após serem identificados foram submetidos a exames necroscópicos convencionais fotodocumentados, utilizando-se a técnica de Ghon modificada, que consiste de abertura do crânio e da cavidade toracoabdominal para remoção dos órgãos em blocos anatômico e funcionalmente relacionados, totalizando quatro blocos (Finkbeiner et al., 2009).

Os órgãos de eleição foram removidos em blocos e examinados detalhadamente, realizando-se o registro fotográfico de alguns deles. Concomitantemente ao exame de necropsia, coletavam-se amostras para análise toxicológica e para o exame convencional histopatológico, independente do tempo de morte.

Para o exame toxicológico, de cada animal foram colhidas amostras em duplicata de fígado, rim e conteúdo estomacal (30 gramas de cada), que eram identificadas, acondicionadas em recipiente plástico estéril e congeladas à $-20^{\circ} \mathrm{C}$. As amostras, quando autorizado pelo tutor do animal ou solicitante do exame necroscópico, eram encaminhadas ao Laboratório de Toxicologia acompanhada de requisição e de atestado de óbito, inclusive com os possíveis agentes suspeitos da intoxicação, para identificação e quantificação de compostos químicos. Uma amostra em duplicata foi mantida em custódia até o resultado oficial desse exame laboratorial, para em seguida ser descartada sob a égide da biossegurança e autorização judicial.

Para a análise histológica, foram coletadas amostras de rim, fígado e encéfalo e fixadas em formalina tamponada a $10 \%$, processadas e as lâminas corados com Hematoxilina e Eosina (HE) para depois serem visualizadas sob microscopia óptica.

Para avaliação das lesões encontradas no encéfalo, rim e fígado, tanto do ponto de vista da macroscopia como da microscopia, foram seguidos critérios utilizados em livros-textos de referência, atentando-se para lesões oriundas da ação de compostos tóxicos, sendo principalmente aquelas que provocam degeneração e outros tipos de lesões reversíveis ou não às células que compõem os tecidos destes órgãos (Jubb et al, 2007; Cummings et al., 2011; Mcgavin e Zachary, 2013).

Por fim, foi realizada análise estatística das distribuições através de frequência absoluta e relativa dos dados obtidos relativo dos casos de intoxicação, considerando-se a espécie acometida, idade, sexo, causa mortis, realização de exame toxicológico e agentes causadores tabulados em MS Excel 2007.

\section{Resultados e discussão}

Foram selecionados 42 casos de animais que atenderam aos critérios de inclusão, no período de 2009 a 2014, conforme ilustrado na Tabela 1.

Tabela 1: Incidência de casos suspeitos de intoxicação em cães e gatos no período de 2009 a 2014

\begin{tabular}{ccc}
\hline Ano & Frequência absoluta & Frequência relativa $(\%)$ \\
\hline 2009 & 5 & 12 \\
2010 & 6 & 14 \\
2011 & 3 & 7 \\
2012 & 10 & 24 \\
2013 & 11 & 26 \\
2014 & 7 & 17 \\
Total & 42 & 100 \\
\hline
\end{tabular}

Dentre os casos, 21 (50\%) foram realizados Boletins de Ocorrência (BO) e $22(52,4 \%)$ foram encaminhados para exame toxicológico. Do total de animais, 31 (73,8\%) eram cães e 11(26,2\%), gatos; quanto ao sexo, do total de 42, $20(47,6 \%)$ fêmeas, $22(52,4 \%)$ machos.

A idade média dos animais acometidos foi de 4,16 anos, sendo que a relação entre sexo, idade, espécie, raça e causa mortis em casos suspeitos de intoxicação criminosa pode ser observada na Tabela 2. 
Foi realizado exame toxicológico em $52 \%$ (22) dos animais, a maior prevalência foi intoxicação por carbamato, correspondendo a $73 \%(16 / 22)$. Organofosforados, piretróides e cumarínicos corresponderam a $9 \%$ (2), 4,5\% (1) e 4,5\% (1) respectivamente. Os demais tiveram resultado negativo no exame toxicológico 9\% (2).

Dentre as causas mortis que constam nos laudos necroscópicos a maioria dos animais veio a óbito devido à insuficiência cardiorrespiratória, sendo choque hipovolêmico a segunda principal causa de morte, no entanto não foi possível obter esta informação de todos os casos.
O conhecimento das ferramentas e dos métodos de investigação é essencial para a compreensão adequada da estrutura e funcionamento das células, tecidos e órgãos. Após a remoção dos fragmentos para avaliação histológica estes devem ser fixados com a finalidade de evitar a digestão dos tecidos por enzimas presentes nas próprias células, fenômeno denominado autólise; e preservar a estrutura e composição molecular dos tecidos. É comum as necropsias forenses e o material histológico encaminhado para exame estar em processo de decomposição, no entanto, uma vez recebido o material o mesmo deve ser

Tabela 2: Descrição dos casos de suspeita de intoxicação criminal de necropsias realizadas em cães e gatos no período de 2009 - 2014

\begin{tabular}{|c|c|c|c|c|c|}
\hline Ano & Sexo & Idade & Espécie & Raça & Causa Mortis \\
\hline 2009 & Fêmea & 5 meses & Canino & SRD & Choque hipovolêmico \\
\hline 2009 & Macho & 2 anos & Felino & SRD & $* *$ \\
\hline 2009 & Fêmea & 3 anos & Canino & Pincher & Insuficiência Cardiorrespiratória \\
\hline 2009 & Fêmea & 10 anos & Canino & Poodle & Insuficiência Cardiorrespiratória \\
\hline 2009 & Macho & 5 meses & Canino & Bull terrier & Insuficiência Cardiorrespiratória \\
\hline 2010 & Fêmea & 2 anos & Canino & Fox Terrier & Insuficiência Cardiorrespiratória \\
\hline 2010 & Macho & 10 anos & Canino & SRD & Choque endotoxêmico \\
\hline 2010 & Macho & 1 ano & Felino & SRD & Choque hipovolêmico \\
\hline 2010 & Macho & 1 ano & Canino & Pastor Alemão & Insuficiência Respiratória aguda \\
\hline 2010 & Macho & 3 anos & Canino & SRD & ** \\
\hline 2010 & Fêmea & 2 anos & Felino & SRD & $* \star$ \\
\hline 2011 & Fêmea & 2,5 anos & Canino & SRD & Insuficiência Cardiorrespiratória \\
\hline 2011 & Fêmea & 3 anos & Canino & SRD & Insuficiência Cardiorrespiratória \\
\hline 2011 & Macho & 3 meses & Felino & SRD & Edema pulmonar \\
\hline 2012 & Macho & 1,5 anos & Canino & Pastor Alemão & Choque hipovolêmico \\
\hline 2012 & Macho & 4,5 anos & Canino & SRD & Insuficiência Cardiorrespiratória \\
\hline 2012 & Fêmea & $\mathrm{N} / \mathrm{l}$ & Canino & SRD & Choque hipovolêmico* \\
\hline 2012 & Macho & 3 anos & Canino & Pastor Alemão & Insuficiência Cardiorrespiratória \\
\hline 2012 & Macho & 13 anos & Canino & Poodle & Insuficiência Cardiorrespiratória \\
\hline 2012 & Macho & 2 anos & Canino & SRD & Insuficiência Cardiorrespiratória \\
\hline 2012 & Fêmea & 1,3 anos & Felino & SRD & $* *$ \\
\hline 2012 & Fêmea & 8 meses & Felino & Siamês & Choque hipovolêmico \\
\hline 2012 & Fêmea & 5 anos & Canino & Pit Bull & Insuficiência Cardiorrespiratória \\
\hline 2012 & Fêmea & 8 meses & Felino & SRD & Insuficiência Cardiorrespiratória \\
\hline 2013 & Fêmea & 12 anos & Canino & SRD & Insuficiência Respiratória Aguda \\
\hline 2013 & Fêmea & 2 anos & Canino & Pinscher & Choque hipovolêmico \\
\hline 2013 & Fêmea & 7 meses & Canino & Lhasa Apso & CID \\
\hline 2013 & Fêmea & $\mathrm{N} / \mathrm{l}$ & Canino & SRD & $* *$ \\
\hline 2013 & Macho & $\mathrm{N} / \mathrm{l}$ & Canino & SRD & ** \\
\hline 2013 & Macho & 10 anos & Felino & SRD & $* *$ \\
\hline 2013 & Macho & 8 meses & Felino & SRD & Insuficiência Respiratória Aguda \\
\hline 2013 & Macho & 13 anos & Canino & Akita & ** \\
\hline 2013 & Macho & 15 anos & Canino & SRD & ** \\
\hline 2013 & Macho & 2 anos & Canino & Pastor Alemão & $* *$ \\
\hline 2013 & Fêmea & 4 anos & Canino & Rottweiller & Insuficiência Respiratória Aguda \\
\hline 2014 & Macho & 10 anos & Canino & Golden & ** \\
\hline 2014 & Fêmea & 3 anos & Canino & Pit Bull & Choque hipovolêmico \\
\hline 2014 & Fêmea & 1 ano & Felino & SRD & Insuficiência Cardiorrespiratória \\
\hline 2014 & Fêmea & 3 anos & Felino & SRD & Insuficiência Cardiorrespiratória \\
\hline 2014 & Macho & 12 anos & Canino & Daschound & Insuficiência Cardiorrespiratória \\
\hline 2014 & Macho & 3 anos & Canino & Basset Hound & Insuficiência Cardiorrespiratória \\
\hline 2014 & Macho & 4 anos & Canino & SRD & Insuficiência Cardiorrespiratória \\
\hline
\end{tabular}


processado, e de qualquer forma é imprescindível a liberação do relatório técnico, se a qualidade prejudicar o resultado, esta informação deve ser ressalvada (Cummings et al., 2011; Tremori e Rocha, 2014).

A análise microscópica de fígado, rim e encéfalo de cães e gatos intoxicados criminalmente apresentou diversas alterações, sendo muitas características de processos degenerativos e fenômenos cadavéricos (Cooper e Cooper, 2008; Cummings et al., 2011).

Nas lesões tóxicas observa-se hiperemia, com estase sanguínea, onde ao corte de órgãos parenquimatosos há aumento de sangue fluindo pela superfície, que também encontra-se mais avermelhada. Microscopicamente muitos vasos, principalmente os capilares, encontram-se congestos (Wang et al., 2007).

Artefatos e alterações post mortem são comuns na avaliação histológica do encéfalo, pois hemorragias decorrentes do método de extração podem ser confundidas com lesões ante mortem, por esta razão é imprescindível o patologista saber diferenciar estas situações para um exame fidedigno e confiável (Finkbeiner et al., 2009).

Um aspecto importante é que quadros clínicos neurológicos provocados por intoxicações são quase sempre fatais, e em algumas situações não há alterações macroscópicas e microscópicas no tecido nervoso, sendo essencial neste caso o patologista recorrer às técnicas toxicológicas (Munro, 1998; Klasseen et al., 2013).

A correlação entre os achados das lesões nos casos de intoxicações criminais em questão condiz com o descrito na literatura, desta forma fortalecendo a importância da Patologia Forense Veterinária para concluir laudos de corpo de delito na perícia veterinária (Tremori e Rocha, 2014).

Os casos de intoxicação criminal são relativamente comuns na rotina médico-veterinária. É importante a capacitação do profissional para estabelecer o diagnóstico definitivo do agente tóxico através de exame toxicológico, que será direcionado de acordo com a interpretação dos achados macroscópicos e microscópicos, desta forma será possível produzir prova pericial que contribuirá judicialmente em crimes envolvendo animais.

Nota-se que houve necropsias brancas ou nulas, quando não é possível determinar a causa mortis, esta é uma situação enfrentada frequentemente pelos legistas, onde o uso de técnicas diagnósticas pode auxiliar na sua elucidação. Por exemplo, o uso de equipamentos de imagem, através da necropsia virtual, pode fornecer achados necroscópicos que não são visualizados no exame convencional, ou ainda a necropsia molecular, que lança mão de análises genéticas para identificar as causas de morte. No entanto, nos casos de intoxicação o exame histopatológico, associado ao exame toxicológico soluciona ao menos 95\% das situações, porém a causa efetiva da morte pode estar mal esclarecida devido a muitas vezes o cadáver encontrar-se em um estado avançado de decomposição, o que é comum em casos forenses.

Verifica-se que a baixa adesão ao exame toxicológico se deve ao fato de que em crimes envolvendo animais não existe um órgão público específico para realização de tais exames, diferentemente do que acontece com seres humanos, no caso o Instituto Médico Legal (IML) é responsável por investigar casos de morte suspeita e morte violenta. Apesar de a legislação brasileira ter mostrado uma evolução com relação às leis de proteção animal, desde a criação da Lei de Crimes Ambientais (9.605/98) onde profere sanções penais e administrativas aos crimes cometidos contra a fauna, ainda não há por parte do governo a iniciativa de elucidar os crimes contra animais. Apesar de existirem exceções, na maioria dos casos, o proprietário como maior interessado é quem deve buscar o auxílio ao setor privado para que a queixa realizada no órgão policial possa ter fundamentação científica através de documentos técnicos e investigação através da instauração de um inquérito.

Esta individualização torna o processo mais exaustivo para o proprietário que muitas vezes, diante de uma situação de fragilidade que é a perda de seu animal de estimação, pode relutar por buscar um serviço privado de Patologia Veterinária para realização do exame necroscópico. Além disso, após ser notificado que terá que encaminhar o material para exame toxicológico de maneira independente e isto poderá resultar um pouco oneroso, também contribui para a baixa adesão ao exame toxicológico.

Não podemos deixar de comentar que nestas situações a cadeia de custódia estará mal preservada, uma vez que será o próprio interessado que irá requisitar e encaminhar o animal para exame necroscópico e toxicológico, tornando fácil o sistema de violação, o que não ocorreria se fosse o caso da realização de todos os procedimentos dentro de um órgão oficial, por exemplo, o Instituto de Criminalística (IC).

Portanto, muitas pessoas deixam de notificar os casos de envolvimentos criminal de animais á autoridade policial, inclusive pela desinformação ou desmotivação que veem ao enfrentar todas as etapas para poder requerer a justiça em prol de crimes contra animais, como podemos perceber nas estatísticas apresentadas no estudo realizado. Este estudo demonstra a necessidade da criação de um Instituto Médico Legal Veterinário, para atender estas solicitações, preservando assim a cadeia de custódia e aumentando o número de casos elucidados envolvendo animais, pois a realização de exames e diagnósticos na perícia veterinária permitem são de extrema importância para a conclusão do laudo pericial ou relatório técnico.

\section{Agradecimentos}

CAPES (Coordenação de Aperfeiçoamento e Desenvolvimento de Pessoal de Nível Superior) Edital Pró Forenses 25/2014 - Brasil.

\section{Referências}

BELOW, E.; LIGNITZ, E. Cases of fatal poisoning in post-mortem examinations at the Institute of Forensic Medicine in Greifswald - analysis of Five decades of post-mortems. Forensic Science International, v.133, p.125-131, 2003.
BERNY, P.; CALONI, F.; CROUBELE, S. et al. Animal poisoning in Europe. Part 2: Companion animals. The Veterinary Journal, v.183, p.255-259, 2010.

BRASIL. Lei $n^{\circ} 69$, de 29 de agosto de 2014. Título IV. Dos crimes contra animais de companhia. Brasília: Diário Oficial da União, 2014. 
BRASIL. Lei n 9.605, de 12 de fevereiro de 1998. Lei de Crimes Ambientais. Brasília: Diário Oficial da União; 1998.

BULCÃO, R.P.; TONELLO, R.; PIVA, R.J. et al. Intoxicação em cães e gatos: diagnóstico toxicológico empregando cromatografia em camada delgada e cromatografia líquida de alta pressão com detecção ultravioleta em amostras estomacais. Ciência Rural, Santa Maria, v.40, n.05, p.1109-1113, 2010.

BYARD, R.W.; BOARDMAN, W. The potential role of forensic pathologists in veterinary forensic medicine. Forensic Science Medicine Pathology, v.7, n.3, p. 231-232, 2011.

COOPER, J.E.; COOPER, M.E. Forensic veterinary medicine: a rapidly evolving discipline. Forensic Science Medicine Pathology, v.4, n.2, p. 75-82, 2008.

CUMMINGS, P.M.; TRELKA, D.P.; SPRINGER, K.M. Poisoning. In:_. Atlas of forensic histopathology. Cambridge University Press, New York, cap. 5, p. 78-83, 2011.

FINKBEINER, W.E.; CONNOLLY, A.; URSELL, P.C.; DAVIS, R.L. Autopsy Pathology: A Manual and Atlas. $2^{a}$ ed. Elsevier Health Sciences, Philadelphia, 2009. 359p.

GWALTNEY-BRANT, S.M. Epidemiology of animal poisoning, Veterinary Toxicology, Elsevier, p.67-73, 2007.

JUBB, K.V.V.F., KENNEDY, P.C., PALMER, N.C. Pathology of Domestic Animals. 5. ed., vol. 1,Edinburgh: Saunders Elsevier, 2007.

KLAASSEN, C.D.; CASARETT, L.J.; DOULL, J.; KLAASEN, C. D. - Casarett and Doull's Toxicology-The basic science of poison. $8^{\circ}$ ed.,New York:McGraw-Hill Education, 2013. 1454p.
MCDONOUGH, S.P.; GERDIN, J.; WUENSCHMANN, A. et al. Illumiating Dark Cases Veterinary Forensic Pathology Emerges. Veterinary Pathology, n.52, v.1, p5-6, 2015.

MCGAVIN M.D.; ZACHARY J.F. Bases da Patologia em Veterinária. 5 ed. São Paulo: Elsevier Editora, 2013. 1344 p.

MEDEIROS, R.J.; MONTEIRO F.O.; SILVA, G.C.; NASCIMENTO A.Jr. Casos de intoxicações exógenas em cães e gatos atendidos na Faculdade de Veterinária da Universidade Federal Fluminense durante o período de 2002 a 2008. Ciência Rural, v.39 n.7, p.2105-2110, 2009.

MUNRO, R. Forensic necropsy. Seminars in Avian and Exotic Pet Medicine, v.7, n.4 (October), pp 201-209, 1998.

TREMORI, T.M.; ROCHA, N.S. O exame do corpo de delito na perícia veterinária (ensaio). Revista de Educação Continuada do CRMV-SP. São Paulo. v.11, n.3, p.30-35, 2013.

WANG, Y.; KRUZIK, P.; HELSBERG, A. et al. Pesticide poisoning in domestic animals and livestock in Austria: $A 6$ years retrospective study. Forensic Science International. v.169, p.157160, 2007.

XAVIER, F.G.; RIGHI, D.A.; SPINOSA, H.S. Fatal poisoning in dogs and cats - 16 - year report in a veterinary pathology service. Brazilian Journal of Veterinary Research and Animal Science, v.44, n.4, p.304-309, 2007. 